\title{
A case-control study on dietary quality indices and glioma
}

\author{
Omid Sadeghi ${ }^{1,2}$, Mehdi Shayanfar ${ }^{3} \dagger$, Minoo Mohammad-Shirazi ${ }^{3}$, Giuve Sharifi ${ }^{4}$ and \\ Ahmad Esmaillzadeh ${ }^{5,6,7 *}$ \\ ${ }^{1}$ Students' Scientific Research Center, Tehran University of Medical Sciences, Tehran, Iran \\ ${ }^{2}$ Department of Community Nutrition, School of Nutritional Sciences and Dietetics, Tebran University of Medical Sciences, \\ Tehran, Iran \\ ${ }^{3}$ Department of Clinical Nutrition and Dietetics, National Nutrition and Food Technology Research Institute, Shabid Beheshti \\ University of Medical Sciences, Tehran, Iran \\ ${ }^{4}$ Department of Neurosurgery, Loghman Hakim Hospital, Shahid Beheshti University of Medical Sciences, Tehran, Iran \\ ${ }^{5}$ Obesity and Eating Habits Research Center, Endocrinology and Metabolism Molecular-Cellular Sciences Institute, Tehran \\ University of Medical Sciences, Tehran, Iran \\ ${ }^{6}$ Department of Community Nutrition, School of Nutritional Sciences and Dietetics, Tebran University of Medical Sciences, \\ Tehran, Iran \\ ${ }^{7}$ Food Security Research Center, Department of Community Nutrition, Isfahan University of Medical Sciences, Isfahan, Iran
}

(Submitted 1 December 2018 - Final revision received 21 March 2019 - Accepted 2 April 2019 - First published online 24 June 2019)

\section{Abstract}

No study is available that has assessed the association of dietary diversity score (DDS) and alternative healthy eating index (AHEI) with glioma. The present study aimed to assess this association in Iranian adults. Overall, 128 pathologically confirmed cases of glioma were enrolled from hospitals and 256 age- and sex-matched controls were recruited from other wards of the hospital between 2009 and 2011. Dietary assessment was done using a validated block-format 123-item semi-quantitative FFQ. Dietary indices including DDS and AHEI-2010 were constructed according to standard methods. After controlling for potential confounders, a significant inverse association was found between DDS and risk of glioma (OR 0.42, $95 \%$ CI 0.19, 0.94). Such finding was also seen when further adjustment was made for BMI; such that participants in the highest quartile of DDS were $56 \%$ less likely to have glioma compared with those in the lowest quartile (OR 0.44, 95\% CI, 0.20, 0.97). In addition, a significant inverse association was found between adherence to AHEI and glioma; such that in the fully adjusted model, participants in the fourth quartile of AHEI had $74 \%$ lower risk of glioma compared with those in the first quartile (OR $0 \cdot 26,95 \%$ CI 0.12, 0.56). In conclusion, we found that greater adherence to the healthy, as measured by AHEI, and diverse, as measured by DDS, diets was associated with decreased odds of glioma.

\section{Key words: Brain tumours: Dietary diversity score: Glioma: Alternative healthy eating index}

Gliomas are the most common primary brain tumour that occurs most frequently in adults and are associated with a high mortality rate $^{(1,2)}$. It accounts for $81 \%$ of brain tumours among adults ${ }^{(1)}$. Although the prevalence of glioma is low around the world, its morbidity and mortality are high ${ }^{(3)}$. National estimates in Iran have shown a mortality rate of 2.92/100 000 in men and 2.46 per 100000 in women ${ }^{(4)}$. Therefore, it is required to find contributing factors in the occurrence and development of glioma.

Dietary intakes are greatly contributing to cancers, including glioma $^{(5)}$. Earlier studies have shown that dietary intake of red meat, salt and alcohol is associated with greater risk of glioma, while intake of fruits, nuts, legumes, vitamin A and antioxidants is associated with decreased odds of brain tumour ${ }^{(5-11)}$. Along with the assessment of link between foods and nutrients with several diseases, assessment of the association with the whole dietary patterns is suggested. Only one case-control study considered dietary patterns in relation to glioma and showed interesting findings ${ }^{(12)}$. Based on this study, high adherence to Dietary Approaches to Stop Hypertension (DASH)style diet was associated with decreased risk of glioma ${ }^{(12)}$. However, other types of dietary pattern or diet quality indices including alternative healthy eating index (AHEI), dietary diversity score (DDS), mean adequacy ratio of nutrients, and dietary energy

\footnotetext{
Abbreviations: AHEI, alternative healthy eating index; DDS, dietary diversity score.

* Corresponding author: Ahmad Esmaillzadeh, email a-esmaillzadeh@sina.tums.ac.ir

$\dagger$ Co-first author: Mehdi Shayanfar
} 
density have not assessed in relation to glioma until now. These indices consider the whole diet rather than its individual components. DDS along with AHEI have been shown as a measure of healthy diet rich in fruits, vegetables and photochemicals ${ }^{(13-15)}$. Although different measures of diet have been linked with cancers, no study examined these indicators in relation to glioma.

Assessing the association between priori defined dietary patterns and glioma is particularly relevant for Middle Eastern countries, because the dietary diversity is low in this region due to low socio-economic status ${ }^{(16)}$. In addition, besides diet other environmental factors are contributing to this condition in the region. Therefore, finding the association of dietary indices such as DDS and AHEI with glioma might present interesting results. The present study aimed to assess these associations among Iranian adults.

\section{Methods}

\section{Sample}

This hospital-based case-control study was carried out on 128 newly diagnosed patients (maximum 1 month elapsed since the detection) and 256 controls without glioma in Tehran, Iran, between 2009 and 2011. Cases were individuals with pathologically confirmed glioma (International Classification of Diseases for Oncology second edition (ICD-O-2) morphology codes 9389481) during the previous month that had been referred to the Neurosurgery department of the hospitals. Controls were apparently healthy individuals who were relatives of patients or those who attended to the outpatients' clinics of the hospital. As we did not examine them in terms of health conditions, they could not be called as absolutely healthy individuals.

\section{Procedure}

Patients with glioma and controls were selected by using convenience-sampling method from the hospitals affiliated to Shahid Beheshti University of Medical Sciences. Cases and controls were matched in terms of age ( \pm 5 years) and sex. Individuals with a history of any type of pathologically confirmed cancer (except glioma), chemotherapy and radiotherapy (due to cancer) were not included in this study. After selection of cases and controls, data on demographic variables, dietary intakes, physical activity and anthropometric measures were collected. Missing information about any variable were completed through contacting subjects. However, in terms of dietary data, we considered unanswered items as null. All cases and controls provided written informed consent. The study was ethically approved by the Medical Ethics Committee of the Tehran University of Medical Sciences, Tehran, Iran.

\section{Assessment of glioma}

Glioma was diagnosed based on pathological test by using ICD-O2 morphology codes $9380-9481^{(17)}$. Only patients with a maximally 1 month of the confirmation of glioma were included in the study.

\section{Variables \\ Dietary assessment}

Usual dietary intakes of participants during a year before the diagnosis of glioma in cases and during a year before the interview in controls were examined by a validated Block-format 123-item semi-quantitative $\mathrm{FFQ}^{(16)}$. The FFQ was consisted of 123 food items with standard portion sizes commonly consumed by Iranian people. Trained interviewers administered the FFQ through face-to-face interviews in the presence of individuals who were involved in the preparation and cooking of foods. All reported consumption frequencies were converted to $\mathrm{g} / \mathrm{d}$ using household measures. Daily intakes of energy and nutrients were computed for each person by using the modified US Department of Agriculture (USDA) food consumption database which was modified for Iranian foods.

Validation study of this FFQ revealed good correlations between dietary intakes assessed by FFQ and those obtained from 24 dietary recalls (two 24-h recalls per month). The reliability of the FFQ was assessed by comparing nutrient intakes obtained from two questionnaires that filled 1 year apart. The correlation coefficients for the reliability of the FFQ for dietary vitamin $\mathrm{E}, \beta$-carotene and vitamin $\mathrm{C}$ were $0.78,0.84$ and $0 \cdot 83^{(18)}$. The energy-adjusted correlation coefficients between the dietary intakes obtained from the FFQ and those from the multiple 24-h dietary recalls were $0.65,0.68$ and 0.65 for vitamin $\mathrm{E}, \beta$-carotene and vitamin $\mathrm{C}^{(16)}$. Overall these data indicated that the FFQ provides reasonably valid measures of the average longtime dietary intakes.

\section{Dietary diversity score}

A method described by Kant et al. was used for scoring dietary diversity ${ }^{(19,20)}$. This method was based on five groups including grains, vegetables, fruits, meats and dairy products, all food groups in the USDA food guide pyramid. The grains group was composed of five components: refined bread, macaroni, whole grain bread, corn flakes and rice. However, in the method of Kant et al., there are seven components in the grains group. As we had no data about intake of biscuits and refined flour, we decided to consider five components. Fruit was defined by summing up fruit and fruit juice, berries and citrus fruits. The story about vegetables was summing up of mixed vegetables, potato, tomato, other starchy vegetables, legumes, yellow vegetables and green vegetables. The group of meat was composed of red meat, poultry, fish and eggs) and the group of dairy products was composed of milk, yoghurt and cheese.

Participants were considered as a 'consumer', and scored as 1, for each components of food groups if they had intakes higher than median levels; otherwise they were given the score of 0 . Then the scores for components in each food group were summed up to have total score of that food group. Then, we divided total scores obtained in each group to the number of components in that group. This value was then multiplied by 2. Total DDS for each participant was then computed by summing up the figures for food groups. For example, in the grains group, if a person had dietary intakes of whole grain bread, macaroni and rice higher than the median values, his or her score was calculated as $(3 / 5) \times 2=1 \cdot 2$. Therefore, the diversity score for the grains group would be 1.2 for that person. After computing the diversity score for the other four groups in that person, total DDS would be computed. Therefore, minimum and maximum scores of total dietary diversity for each participant were between 0 and 10 . 


\section{Alternative healthy eating index}

We calculated AHEI-2010 using information obtained from FFQ. The AHEI incorporates several aspects of the original HEI designed by Kennedy et al. ${ }^{(21-23)}$. This method considered eleven components including fruit, vegetables, whole grains, nuts and legumes, long-chain $n-3$ fats (DHA and EPA), PUFA, wine consumption, sugar-sweetened drinks and fruit juice, red and processed meats, trans-fat and $\mathrm{Na}$ intake. Due to lack of data on wine consumption and trans-fat in our database, we considered nine components to compute AHEI in the present study. Individuals in the highest decile of whole grains, vegetables, fruits, nuts and legumes, PUFA and long-chain $n-3$ fats were given the score of 10 and those in the lowest decile received the score of 1 . Individuals in other deciles received the corresponding scores. In contrast, individuals with the highest intake of sugar-sweetened drinks and fruit juice, red and processed meats, and $\mathrm{Na}$ were given the score of 1 and those with the lowest consumption of these components received the score of 10 . Total AHEI score for each participant was then computed by summing up the scores for these ten components. It was varied from 9 to 81 .

\section{Assessment of other variables}

A pretested questionnaire was used to collect data on age, sex, marital status, place of residence, education, occupation, smoking status, use of supplements, family history of cancers and glioma, history of allergy and trauma, history of hypertension, exposure to chemicals in the past 10 years, cooking methods (barbecue/microwave/canned foods/fried foods), drug use, personal hair dye use, duration of cell phone use and history of exposure to radiographic X-rays. International Physical Activity Questionnaire (IPAQ) was used for measuring physical activity of participants through face to face interview. All results of the IPAQ were expressed as metabolic equivalents per week (MET/week). Body weight was quantified by digital scale to the nearest $500 \mathrm{~g}$ with the subjects wearing the light clothing and no shoes. Height was measured by a tape measure to the nearest 0.5 $\mathrm{cm}$ in standing status while the subject's shoulders were in normal position. BMI was calculated by dividing weight in $\mathrm{kg}$ by height in $\mathrm{m}$ squared. All measurements were completed by a trained dietitian.

On the basis of previous studies, we considered farmers as those having a high-risk occupation for glioma ${ }^{(24)}$. Individuals who lived in places near the electromagnetic fields and cell phone and broadcast antennas in the last 10 years were defined as living in high-risk areas ${ }^{(25)}$. Individuals who consumed fried foods at least twice per week were considered as frequent fried food users. This definition was also used for barbecue use, microwave use as well as for consumption of canned foods.

\section{Analysis plan}

Cases and controls were compared in terms of general characteristics and dietary intakes using independent-samples $t$ test and $\chi^{2}$, where appropriate. After construction of DDS and AHEI, as mentioned above, we obtained energy-adjusted scores of DDS and AHEI using residual method ${ }^{(26)}$. Then, participants were categorised based on quartile cut-off points of DDS and AHEI that were obtained from the control group. We used one-way ANOVA and $\chi^{2}$ tests to assess continuous and categorical variables across quartiles of DDS and AHEI. Binary logistic regression in different models was applied to evaluate the association of DDS and AHEI with glioma. First, we included all confounders into models and then, we retained those variables that had a significant contribution to glioma. In the first model, we controlled for energy intake (continues). Further adjustments were made for family history of glioma (yes/no), marital status (married/single/divorced), high-risk occupation (farmer/non-farmer), highrisk residential area (yes/no), supplement use (yes/no), history of exposure to radiographic X-rays (yes/no), history of head trauma (yes/no), smoking (smoker/non-smoker), drug use (yes/no), personal hair dye use (yes/no), frequent fried food intake (yes/no) and frequent use of microwave (yes/no) in the second model. Additional controlling was performed for BMI (continues) in the last model. All confounders were chosen based on earlier publications ${ }^{(27-30)}$. In these analyses, the first quartile of DDS and AHEI was considered as the reference category. To obtain the overall trend of OR across increasing quartiles of DDS and AHEI, we considered these quartiles as an ordinal variable in the logistic regression models. All statistical analyses were conducted using SPSS software (version 19.0; SPSS Inc). $P$ values less than 0.05 was considered as significant level.

\section{Results}

General characteristics and dietary intakes of cases and controls are presented in Table 1. Cases were more likely to have highrisk jobs, history of exposure to radiographic X-rays, history of head trauma, family history of glioma and history of exposure to chemicals and live in high-risk residential areas than controls. Cases were also more likely to be frequent fried food users and had higher intakes of red meat, $\mathrm{Na}$, and refined and whole grains than controls. Controls were more likely to be current smokers, hair dye users, supplement users, microwave users and have a long duration of cell phone use than cases. Controls had higher intakes of Ca, PUFA, fruits, dairy products, salt, legumes and nuts, and higher scores of AHEI compared with cases.

General characteristics of participants across quartiles of DDS and AHEI scores are shown in Table 2. Individuals in the top quartile of DDS were less likely to be a current smokers and have a high-risk job compared with those in the bottom quartile. No other significant difference was found in terms of general characteristics across quartiles of DDS. In addition, compared with participants in the lowest quartile of AHEI, those in the highest quartile were more likely to be female, older, married, have a high-risk job and history of allergy, use hair colour, and less likely to be a current smoker, frequently exposed to microwave, use cell phone, frequently use fried foods and barbecue. We found no other significant difference in terms of general characteristics across quartiles of AHEI.

Dietary intakes of cases and controls across quartiles of DDS and AHEI scores are presented in Table 3. Those in the top 
Table 1. General characteristics and dietary intakes of cases and controls ( $n$ 384) (Mean values and standard deviations; percentages)

\begin{tabular}{|c|c|c|c|c|c|}
\hline & \multicolumn{2}{|c|}{ Cases ( $n$ 128) } & \multicolumn{2}{|c|}{ Controls ( $n$ 256) } & \multirow[b]{2}{*}{$P^{*}$} \\
\hline & Mean & SD & Mean & SD & \\
\hline Age (years) & $43 \cdot 4$ & $14 \cdot 6$ & $42 \cdot 8$ & $13 \cdot 3$ & 0.65 \\
\hline Males (\%) & \multicolumn{2}{|c|}{$58 \cdot 6$} & \multicolumn{2}{|c|}{$58 \cdot 2$} & 0.94 \\
\hline Weight $(\mathrm{kg})$ & $74 \cdot 6$ & 13.7 & $72 \cdot 1$ & $12 \cdot 1$ & 0.07 \\
\hline BMI $\left(\mathrm{kg} / \mathrm{m}^{2}\right)$ & $26 \cdot 3$ & $4 \cdot 3$ & $26 \cdot 1$ & 3.8 & 0.76 \\
\hline Married (\%) & \multicolumn{2}{|c|}{78.9} & \multicolumn{2}{|c|}{$80 \cdot 1$} & 0.66 \\
\hline University graduated (\%) & \multicolumn{2}{|c|}{$11 \cdot 7$} & \multicolumn{2}{|c|}{$16 \cdot 8$} & 0.19 \\
\hline High-risk jobs†(\%) & \multicolumn{2}{|c|}{$10 \cdot 2$} & \multicolumn{2}{|c|}{$2 \cdot 7$} & 0.01 \\
\hline High-risk residential areał (\%) & \multicolumn{2}{|c|}{30.5} & \multicolumn{2}{|c|}{21.5} & 0.05 \\
\hline Duration of cell phone use (years) & $2 \cdot 8$ & $2 \cdot 9$ & 3.7 & $2 \cdot 6$ & 0.01 \\
\hline History of exposure to radiographic X-rays (\%) & \multicolumn{2}{|c|}{$15 \cdot 6$} & \multicolumn{2}{|c|}{$7 \cdot 4$} & 0.01 \\
\hline History of head trauma (\%) & \multirow{2}{*}{\multicolumn{2}{|c|}{$\begin{array}{l}43.8 \\
25.0\end{array}$}} & \multicolumn{2}{|c|}{28.9} & 0.01 \\
\hline History of allergy (\%) & & & \multicolumn{2}{|c|}{$29 \cdot 3$} & 0.37 \\
\hline History of hypertension (\%) & \multicolumn{2}{|c|}{$2 \cdot 3$} & & & 0.21 \\
\hline History of dental photography (\%) & \multicolumn{2}{|c|}{$46 \cdot 1$} & & & 0.02 \\
\hline Current smoker & & & & & 0.01 \\
\hline Frequent fried food intakes§ (\%) & & & & & 0.01 \\
\hline Frequent use of barbecue\| (\%) & & & & & 0.34 \\
\hline Frequent microwave use $\|(\%)$ & & & & & 0.01 \\
\hline Frequent canned foods intake $\|(\%)$ & & & & & 0.88 \\
\hline Drug use (\%) & & & & & 0.29 \\
\hline Personal hair dye use (\%) & & & & & $<0.001$ \\
\hline Exposure to chemicals (\%) & & & & & 0.01 \\
\hline Family history of glioma (\%) & & & & & $<0.001$ \\
\hline Family history of cancer (\%) & & & & & 0.82 \\
\hline Supplement use (\%) & & & & & 0.03 \\
\hline Physical activity (MET-h/d) & $34 \cdot 8$ & $6 \cdot 3$ & 33.8 & $6 \cdot 3$ & 0.13 \\
\hline Energy $(\mathrm{kcal} / \mathrm{d}) \emptyset$ & 2580 & 560 & 2561 & 722 & 0.79 \\
\hline Nutrient intakes & & & & & \\
\hline Proteins $(\mathrm{g} / \mathrm{d})$ & $98 \cdot 2$ & $21 \cdot 7$ & $97 \cdot 1$ & $29 \cdot 7$ & 0.70 \\
\hline Fats $(\mathrm{g} / \mathrm{d})$ & $61 \cdot 8$ & $18 \cdot 6$ & $66 \cdot 1$ & $21 \cdot 6$ & 0.05 \\
\hline SFA $(g / d)$ & $19 \cdot 1$ & $7 \cdot 2$ & $20 \cdot 7$ & $9 \cdot 0$ & 0.09 \\
\hline PUFA $(g / d)$ & $12 \cdot 6$ & $3 \cdot 7$ & 13.6 & $5 \cdot 0$ & 0.02 \\
\hline Long-chain fatty acids (mg/d) & $17 \cdot 5$ & $29 \cdot 7$ & $16 \cdot 3$ & 23.4 & 0.67 \\
\hline $\mathrm{Ca}(\mathrm{mg} / \mathrm{d})$ & 1021 & 260 & 1122 & 321 & 0.01 \\
\hline Dietary fibre $(\mathrm{g} / \mathrm{d})$ & 23.4 & 11.2 & 23.0 & $14 \cdot 2$ & 0.82 \\
\hline $\mathrm{Na}(\mathrm{mg} / \mathrm{d})$ & $4822 \cdot 8$ & $1506 \cdot 1$ & $4149 \cdot 4$ & $1768 \cdot 4$ & $<0.001$ \\
\hline Food groups $(\mathrm{g} / \mathrm{d})$ & & & & & \\
\hline Refined grains & $501 \cdot 2$ & 174.7 & 421.0 & $182 \cdot 3$ & $<0.001$ \\
\hline Whole-grains & $176 \cdot 8$ & $134 \cdot 0$ & $150 \cdot 0$ & $108 \cdot 2$ & 0.04 \\
\hline White meats & $30 \cdot 1$ & $13 \cdot 6$ & $32 \cdot 6$ & 22.5 & 0.24 \\
\hline Red meats & 41.4 & $27 \cdot 8$ & $36 \cdot 0$ & $19 \cdot 8$ & 0.03 \\
\hline Fish & 9.2 & $12 \cdot 1$ & 9.0 & 9.1 & 0.88 \\
\hline Fruits & $325 \cdot 3$ & $99 \cdot 7$ & $360 \cdot 8$ & $124 \cdot 2$ & 0.01 \\
\hline Vegetables & 257.8 & $82 \cdot 6$ & $274 \cdot 2$ & $86 \cdot 2$ & 0.08 \\
\hline Dairy products & $309 \cdot 2$ & $116 \cdot 7$ & $355 \cdot 0$ & 131.5 & 0.01 \\
\hline Legumes and nuts & $40 \cdot 6$ & $22 \cdot 7$ & $46 \cdot 0$ & $20 \cdot 0$ & 0.02 \\
\hline SSB & $79 \cdot 1$ & $67 \cdot 3$ & 83.5 & 74.5 & 0.57 \\
\hline Salt & $5 \cdot 7$ & $2 \cdot 0$ & $6 \cdot 3$ & $2 \cdot 1$ & 0.01 \\
\hline DDS scores & 4.8 & 1.5 & $5 \cdot 1$ & 1.9 & 0.11 \\
\hline AHEI scores & $46 \cdot 5$ & $10 \cdot 0$ & 50.9 & $9 \cdot 2$ & $<0.001$ \\
\hline
\end{tabular}

MET, metabolic equivalents; SSB, sugar-sweetened beverages; DDS, dietary diversity score; AHEI, alternative healthy eating index.

* Obtained from independent-samples $t$ test or $\chi^{2}$ test, where appropriate.

†Farmers were considered as having a high-risk occupation.

¥ Individuals who lived in places near electromagnetic fields and cell phone and broadcast antennas in the last 10 years were defined as living in high-risk areas.

$\S$ Individuals who consumed fried food at least twice per week considered as frequent fried food users.

|| Individuals who used barbecue, microwave and canned foods at least twice per week were considered as frequent users.

१ी To convert kcal to kJ, multiply by 4.184.

quartile of DDS had higher intakes of fruit, vegetables, fish, legumes and nuts, dairy products, protein, fat, SFA and Ca compared with individuals in the bottom quartile. In addition, compared with participants in the first quartile of AHEI, those in the fourth quartile had higher intakes of fruit, vegetables, dairy products, legumes and nuts, whole grains, fat, protein, PUFA and $\mathrm{Ca}$ and lower intakes of red meat, refined grains, salt, sugar-sweetened beverages and $\mathrm{Na}$. No other significant difference was found in terms of dietary intakes across quartiles of DDS and AHEI scores.

Multivariable-adjusted OR and 95\% CI for glioma across quartiles of DDS and AHEI are shown in Table 4. After controlling for energy intake and other potential confounders, a significant inverse association was found between DDS and risk of 
Table 2. General characteristics of study participants cross quartiles (Q) of dietary diversity score (DDS) and alternative healthy eating index (AHEI) scores ( $n 384$ ) (Mean values and standard deviations; percentages)

\begin{tabular}{|c|c|c|c|c|c|c|c|c|c|c|c|c|c|c|c|c|c|}
\hline & \multicolumn{9}{|c|}{ Quartiles of DDS } & \multicolumn{8}{|c|}{ Quartiles of AHEI } \\
\hline & \multicolumn{2}{|c|}{ Q1 } & \multicolumn{2}{|c|}{ Q2 } & \multicolumn{2}{|c|}{ Q3 } & \multicolumn{2}{|c|}{ Q4 } & \multirow[b]{2}{*}{$P^{*}$} & \multicolumn{2}{|c|}{ Q1 } & \multicolumn{2}{|c|}{ Q2 } & \multicolumn{2}{|c|}{ Q3 } & Q4 & \multirow[b]{2}{*}{$P^{*}$} \\
\hline & Mean & SD & Mean & SD & Mean & SD & Mean & SD & & Mean & SD & Mean & SD & Mean & SD & Mean SD & \\
\hline Cases (\%) & \multicolumn{2}{|c|}{34.7} & \multicolumn{2}{|c|}{43.9} & \multicolumn{2}{|c|}{$28 \cdot 1$} & \multicolumn{2}{|c|}{22.9} & 0.01 & \multicolumn{2}{|c|}{47.5} & \multicolumn{2}{|c|}{33.3} & \multicolumn{2}{|c|}{17.9} & $27 \cdot 3$ & $<0.001$ \\
\hline Age (years) & 42 & $14 \cdot 5$ & 43.9 & $13 \cdot 6$ & $42 \cdot 5$ & 13.9 & $43 \cdot 2$ & $12 \cdot 8$ & 0.76 & $40 \cdot 4$ & $15 \cdot 0$ & $43 \cdot 1$ & $14 \cdot 0$ & $42 \cdot 6$ & $13 \cdot 1$ & $46 \cdot 6 \quad 11.2$ & 0.01 \\
\hline Females (\%) & \multicolumn{2}{|c|}{$36 \cdot 7$} & \multicolumn{2}{|c|}{47.4} & \multicolumn{2}{|c|}{$39 \cdot 3$} & \multicolumn{2}{|c|}{$42 \cdot 2$} & 0.43 & \multicolumn{2}{|c|}{34.4} & \multicolumn{2}{|c|}{37.5} & 35 & & 61.4 & $<0.001$ \\
\hline $\mathrm{BMI}\left(\mathrm{kg} / \mathrm{m}^{2}\right)$ & $26 \cdot 2$ & $4 \cdot 6$ & $25 \cdot 8$ & 3.8 & $26 \cdot 4$ & 4 & $26 \cdot 2$ & 3.3 & 0.74 & $26 \cdot 2$ & 3.9 & $25 \cdot 9$ & 4.0 & $25 \cdot 9$ & 3.8 & $26 \cdot 5$ & 0.78 \\
\hline Married (\%) & 72 & & 76 & & 84 & & 88 & & 0.05 & 70 & & 79 . & & 78. & & $94 \cdot 3$ & $<0.001$ \\
\hline University graduated (\%) & $1<$ & & 17 & & 11 & & 16 & & 0.61 & 14 & & 14. & & 12. & & $18 \cdot 2$ & 0.80 \\
\hline High-risk job† (\%) & 10 & & & 3 & 3 & 4 & 1. & $\cdot 2$ & 0.04 & 4 & $\cdot 1$ & 6 . & 3 & 0 & & $10 \cdot 2$ & 0.02 \\
\hline High-risk job residential area $(\%)$ & 2 & & 21 & .9 & 27 & & 28 & & 0.55 & 25 & & 24. & & 29. & & $19 \cdot 3$ & 0.49 \\
\hline Duration of cell phone use (years) & 3.4 & $2 \cdot 4$ & $3 \cdot 2$ & $2 \cdot 7$ & 3.5 & $2 \cdot 8$ & 3.5 & $2 \cdot 7$ & 0.86 & 3.5 & $2 \cdot 4$ & 3.7 & $2 \cdot 6$ & 3.7 & $2 \cdot 8$ & $2 \cdot 8$ & 0.02 \\
\hline $\begin{array}{l}\text { History of exposure to radiographic } \\
\text { X-rays }(\%)\end{array}$ & 10 & & & 1 & 14 & & 10 & & 0.26 & 15 & & 8. & & 5 . & 1 & $9 \cdot 1$ & 0.08 \\
\hline History of head trauma (\%) & 33 & & 35 & & 32 & & 33 & & 0.98 & 34 & & 28. & & 41. & & 33.0 & 0.35 \\
\hline History of allergy (\%) & 22 & & 32 & & 27 & & 26 & & 0.60 & 17 & & 33 . & & 32. & & 33.0 & 0.01 \\
\hline f hypertension & & & & 6 & 6 & 7 & 3. & 6 & 0.52 & 3 & 3 & 2 . & $\cdot 1$ & 5 . & 1 & $6 \cdot 8$ & 0.38 \\
\hline Smoking s & 31 & & 17 & & 22 & & 15 & & 0.03 & 29 & & 27. & & 19. & & $8 \cdot 0$ & 0.001 \\
\hline Frequent fried food intake§ (\%) & 8 & & 82 & & 84 & & 80 & & 0.93 & 88 & & 86. & & 71. & & 78.4 & 0.01 \\
\hline Frequent use of barbecue $\|(\%)$ & 13 & & & 9 & 18 & & 15 & & 0.17 & 20 & & 10. & & 12 . & & $6 \cdot 8$ & 0.02 \\
\hline Frequent microwave use $\|(\%)$ & 13 & & 1 & & 15 & & 19 & & 0.68 & 14 & & 15 . & & 19. & & $12 \cdot 5$ & 0.68 \\
\hline Frequent canned foods intake\| (\%) & & & & 5 & 7 & 9 & 7. & 2 & 0.56 & 6 & 6 & 5 . & $\cdot 2$ & 7. & & 4.5 & 0.82 \\
\hline Drug & & & & 5 & 7 & 9 & 8 & 4 & 0.42 & 4 & $\cdot 1$ & 9 & 4 & 7. & 7 & 3.4 & 0.24 \\
\hline Personal hair dye use (\%) & 30 & & 32 & & 38 & & 38 & & 0.57 & 21 & & 34 & & 35 & & $52 \cdot 3$ & $<0.001$ \\
\hline Exposure to chemicals (\%) & $1<$ & & 11 & & 13 & & 15 & & 0.84 & 16 & & 10 & & 10 & & $15 \cdot 9$ & 0.42 \\
\hline Family history of glioma (\%) & 11 & & 13 & & 6 & & 8 & 4 & 0.44 & 13 & & 9 & 4 & 6. & 4 & $10 \cdot 2$ & 0.48 \\
\hline Family history of cancer (\%) & & & 34 & & 40 & & 34 & & 0.18 & 31 & & 28 & & 41 & & $36 \cdot 4$ & 0.27 \\
\hline Supplem & 10 & & 10 & & 15 & & 16 & & 0.39 & 13 & & 10 & & 12. & & $14 \cdot 8$ & 0.82 \\
\hline Physical activity (MET) & $34 \cdot 3$ & 6.5 & 33.8 & 5.5 & $34 \cdot 3$ & 5.7 & $34 \cdot 1$ & $5 \cdot 3$ & 0.87 & 33.9 & $5 \cdot 7$ & 33.5 & $5 \cdot 3$ & $34 \cdot 0$ & 6.0 & $35 \cdot 0$ & 0.34 \\
\hline
\end{tabular}

MET, metabolic equivalents.

* Obtained from ANOVA or $\chi^{2}$ test, where appropriate.

$\dagger$ Farmers were considered as having a high-risk occupation.

¥ Individuals who lived in places near electromagnetic fields and cell phone and broadcast antennas in the last 10 years were defined as living in high-risk areas.

$\S$ Individuals who consumed fried food at least twice per week considered as frequent fried food users.

|| Individuals who used barbecue, microwave and canned foods at least twice per week were considered as frequent users.

Table 3. Dietary and nutrient intakes of study participants across quartiles (Q) of dietary diversity score (DDS) and alternative healthy eating index (AHEI) scores $(n 384) \dagger$ (Mean values with their standard errors; percentages)

\begin{tabular}{|c|c|c|c|c|c|c|c|c|c|c|c|c|c|c|c|c|c|c|}
\hline & \multicolumn{9}{|c|}{ Quartiles of DDS } & \multicolumn{9}{|c|}{ Quartiles of AHEI } \\
\hline & \multicolumn{2}{|c|}{ Q1 } & \multicolumn{2}{|c|}{ Q2 } & \multicolumn{2}{|c|}{ Q3 } & \multicolumn{2}{|c|}{ Q4 } & \multirow[b]{2}{*}{$P^{*}$} & \multicolumn{2}{|c|}{ Q1 } & \multicolumn{2}{|c|}{ Q2 } & \multicolumn{2}{|c|}{ Q3 } & \multicolumn{2}{|c|}{ Q4 } & \multirow[b]{2}{*}{$P^{*}$} \\
\hline & Mean & SE & Mean & SE & Mean & SE & Mean & $\mathrm{SE}$ & & Mean & SE & Mean & SE & Mean & SE & Mean & SE & \\
\hline Cases (\%) & \multicolumn{2}{|c|}{34.7} & \multicolumn{2}{|c|}{43.9} & \multicolumn{2}{|c|}{$28 \cdot 1$} & \multicolumn{2}{|c|}{22.9} & 0.01 & \multicolumn{2}{|c|}{47.5} & \multicolumn{2}{|c|}{33.3} & \multicolumn{2}{|c|}{17.9} & \multicolumn{2}{|c|}{$27 \cdot 3$} & $<0.001$ \\
\hline Food groups $(\mathrm{g} / \mathrm{d})$ & & & & & & & & & & & & & & & & & & \\
\hline Fruits & 263.9 & 11.4 & $327 \cdot 3$ & 8.3 & 393.9 & 11.0 & 431.6 & $10 \cdot 2$ & $<0.001$ & $308 \cdot 1$ & 8.4 & $298 \cdot 3$ & 9.0 & 395.4 & $15 \cdot 3$ & $419 \cdot 4$ & 11.6 & $<0.001$ \\
\hline Vegetables & $219 \cdot 2$ & 7.8 & 252.6 & 6.4 & 299.6 & 9.6 & 315.9 & 7.7 & $<0.001$ & $230 \cdot 0$ & 5.4 & $248 \cdot 3$ & 6.1 & 288.4 & $11 \cdot 0$ & 330.6 & 9.4 & $<0.001$ \\
\hline Red meat & 32.5 & 2.4 & 30.9 & 1.4 & 33.6 & 1.5 & 35.2 & 1.4 & 0.38 & 38.9 & 1.9 & 30.4 & 1.5 & 31.8 & 1.7 & 28.2 & 1.4 & $<0.001$ \\
\hline Fish & 6.6 & 0.9 & 8.4 & 1.0 & 10.5 & 1.1 & 11.6 & 1.0 & 0.005 & 8.0 & 0.8 & 9.8 & 1.0 & 8.4 & $1 \cdot 2$ & 10.4 & 1.1 & 0.30 \\
\hline Dairy & 282.5 & 13.4 & 327.0 & $10 \cdot 3$ & 362.6 & 13.9 & $400 \cdot 2$ & $12 \cdot 1$ & $<0.001$ & $333 \cdot 3$ & 11.8 & $305 \cdot 6$ & 11.8 & 355.1 & 15.7 & $372 \cdot 1$ & 12.8 & 0.003 \\
\hline Legumes and $n$ & 36.6 & 1.9 & $42 \cdot 7$ & 1.9 & 48.4 & 2.3 & 50.5 & $2 \cdot 1$ & $<0.001$ & 32.5 & 1.2 & $40 \cdot 1$ & 1.9 & 52.4 & 2.5 & $57 \cdot 2$ & $2 \cdot 1$ & $<0.001$ \\
\hline Whole grains & $175 \cdot 3$ & 13.8 & 148 & $10 \cdot 6$ & $156 \cdot 3$ & 11.9 & 157.3 & 11.4 & 0.40 & $115 \cdot 0$ & 8.9 & $154 \cdot 1$ & 11.4 & $182 \cdot 3$ & $12 \cdot 8$ & $204 \cdot 3$ & 13.9 & $<0.001$ \\
\hline Refined grains & 448.6 & 19.2 & 435.6 & 15.6 & 458.6 & 19.2 & $451 \cdot 3$ & 21.9 & 0 & $510 \cdot 3$ & 17.5 & $448 \cdot 0$ & $15 \cdot 2$ & 432.4 & $22 \cdot 4$ & 373.9 & 17.1 & $<0.001$ \\
\hline Salt & $6 \cdot 1$ & 0.2 & $6 \cdot 1$ & 0.1 & $6 \cdot 3$ & 0.2 & 5.8 & 0.2 & 0.58 & $6 \cdot 2$ & 0.1 & 6.4 & 0.2 & $6 \cdot 3$ & 0.2 & 5.4 & 0.2 & 0.003 \\
\hline SSB & 66.8 & $6 \cdot 2$ & $81 \cdot 7$ & $6 \cdot 1$ & 87.5 & $7 \cdot 3$ & $94 \cdot 3$ & 9.9 & 0.0 & 11.8 & $7 \cdot 0$ & 79.2 & 7.9 & 79.7 & 7.0 & 45.8 & 4.5 & $<0.001$ \\
\hline \multicolumn{19}{|l|}{ Nutrients } \\
\hline Energy $(\mathrm{kcal} / \mathrm{d}) \ddagger$ & 2557.4 & $86 \cdot 3$ & $2450 \cdot 4$ & 48.4 & 2649.7 & 72.9 & 2652.7 & 63.2 & 0.10 & 558.8 & $62 \cdot 9$ & $2461 \cdot 2$ & $55 \cdot 0$ & 2673.6 & 98.5 & $2602 \cdot 1$ & 58.6 & 0.20 \\
\hline Protein $(\mathrm{g} / \mathrm{d})$ & 93.8 & 3.6 & 94.1 & 1.9 & $100 \cdot 5$ & 2.8 & $102 \cdot 8$ & 2.4 & 0.04 & 93.6 & 1.9 & 94.3 & 2.0 & $104 \cdot 4$ & 4.7 & 99.8 & $2 \cdot 4$ & 0.02 \\
\hline Fat $(\mathrm{g}$ & 60.6 & $2 \cdot 3$ & 61.5 & 1.8 & $67 \cdot 4$ & $2 \cdot 1$ & 70.8 & 1.9 & 0.001 & 61.2 & $1 \cdot 7$ & $61 \cdot 2$ & 1.9 & $66 \cdot 7$ & $2 \cdot 8$ & $71 \cdot 3$ & 1.9 & 0.001 \\
\hline Carbohydrate (g/d) & 424.9 & 16.7 & 397.1 & 8.3 & $427 \cdot 8$ & $12 \cdot 1$ & 419.9 & 10.5 & 0.22 & $423 \cdot 4$ & 12.8 & 398.7 & 9.5 & $430 \cdot 6$ & 14.8 & $412 \cdot 7$ & 10.5 & 0.30 \\
\hline SFA (g/d) & 18.9 & 1.0 & $19 \cdot 3$ & 0.7 & 21.3 & 0.8 & 21.9 & 0.9 & 0.04 & $19 \cdot 6$ & 0.6 & 19.3 & 0.8 & 20.9 & 1.1 & 21.5 & 0.9 & 0.25 \\
\hline PUF & $12 \cdot 4$ & 0.4 & $12 \cdot 7$ & 0.4 & 13.4 & 0 & $15 \cdot 0$ & 0 & & 11.6 & 0.3 & $12 \cdot 6$ & 0 & $13 \cdot 7$ & 0.5 & 15.9 & 0.5 & $<0.001$ \\
\hline LC-FA (mg/d) & $18 \cdot 3$ & 4.1 & 13.2 & 1.2 & $17 \cdot 0$ & $2 \cdot 1$ & 19.4 & $2 \cdot 2$ & 0.34 & $15 \cdot 9$ & 2.6 & $15 \cdot 2$ & 1.7 & 20.9 & 3.9 & 15.8 & 1.9 & 0.45 \\
\hline $\mathrm{Ca}(\mathrm{g} / \mathrm{d})$ & 979.6 & 38.3 & 1072.5 & $32 \cdot 3$ & $1155 \cdot 9$ & 33.9 & $1216 \cdot 1$ & 27.5 & $<0.001$ & $1065 \cdot 3$ & 24.7 & $1027 \cdot 4$ & 30.9 & 1211.1 & $56 \cdot 2$ & 1118.9 & 27.2 & 0.002 \\
\hline $\mathrm{Na}(\mathrm{mg} / \mathrm{c}$ & $4180 \cdot 8$ & 134 & $4484 \cdot 1$ & 143 & $4702 \cdot 2$ & 183 & $4639 \cdot 1$ & 203 & 0.12 & $5079 \cdot 2$ & 143 & 4332.7 & 152 & 4518.4 & 194 & $3791 \cdot 2$ & 143 & $<0.001$ \\
\hline Dietary fibre $(\mathrm{g} / \mathrm{d})$ & 20.9 & 1.5 & 22.9 & 1.5 & 24.4 & 1.2 & 24.5 & 0.9 & 0.23 & $22 \cdot 3$ & $1 \cdot 0$ & 21.7 & 1.4 & $25 \cdot 2$ & $2 \cdot 1$ & 23.8 & 0.8 & 0.33 \\
\hline
\end{tabular}

SSB, sugar-sweetened beverages; LC-FA, long-chain fatty acids.

* Obtained from ANOVA.

$\dagger$ All values, except energy intake, are energy-adjusted.

‡ To convert kcal to kJ, multiply by 4.184. 
Table 4. Risk for glioma according to quartiles (Q) of dietary diversity score (DDS) and alternative healthy eating index (AHEI) scores $(n 384)$ (Odds ratios and $95 \%$ confidence intervals)

\begin{tabular}{|c|c|c|c|c|c|c|c|c|c|c|c|c|c|c|c|c|}
\hline & \multicolumn{7}{|c|}{ Quartiles of DDS } & \multirow[b]{3}{*}{$P_{\text {trend }}$} & \multicolumn{7}{|c|}{ Quartiles of AHEI } & \multirow[b]{3}{*}{$P_{\text {trend }}$} \\
\hline & \multirow{2}{*}{$\frac{\mathrm{Q} 1}{\mathrm{OR}}$} & \multicolumn{2}{|r|}{ Q2 } & \multicolumn{2}{|r|}{ Q3 } & \multicolumn{2}{|r|}{ Q4 } & & \multirow{2}{*}{$\frac{\mathrm{Q} 1}{\mathrm{OR}}$} & \multicolumn{2}{|r|}{ Q2 } & \multicolumn{2}{|r|}{ Q3 } & \multicolumn{2}{|r|}{ Q4 } & \\
\hline & & OR & $95 \% \mathrm{Cl}$ & OR & $95 \% \mathrm{Cl}$ & OR & $95 \% \mathrm{Cl}$ & & & OR & $95 \% \mathrm{Cl}$ & OR & $95 \% \mathrm{Cl}$ & OR & $95 \% \mathrm{Cl}$ & \\
\hline Crude & 1 & 1.47 & $0.84,2.56$ & 0.73 & $0.39,1.36$ & 0.55 & $0.28,1.08$ & 0.02 & 1 & 0.55 & $0.31,0.96$ & 0.24 & $0.12,0.47$ & 0.41 & $0.23,0.74$ & $<0.001$ \\
\hline Model $1^{*}$ & 1 & 1.49 & $0.85,2.60$ & 0.72 & $0.39,1.35$ & 0.55 & $0.28,1.07$ & 0.02 & 1 & 0.55 & $0.32,0.96$ & 0.23 & $0.12,0.47$ & 0.41 & $0.22,0.74$ & $<0.001$ \\
\hline Model $2 \dagger$ & 1 & 1.33 & $0.69,2.58$ & 0.67 & $0.32,1.37$ & 0.42 & $0.19,0.94$ & 0.01 & 1 & 0.47 & $0.24,0.91$ & 0.19 & $0.08,0.42$ & 0.25 & $0.12,0.55$ & $<0.001$ \\
\hline Model 3ł & 1 & 1.36 & $0.70,2.64$ & 0.68 & $0.33,1.41$ & 0.44 & $0.20,0.97$ & 0.01 & 1 & 0.48 & $0.25,0.93$ & 0.19 & $0.08,0.43$ & 0.26 & $0.12,0.56$ & $<0.001$ \\
\hline
\end{tabular}

* Model 1: adjusted for energy intake.

† Model 2: additionally adjusted for family history of glioma, marital status, high-risk occupation, high-risk residential area, supplement use, history of exposure to radiographic X-rays, history of head trauma, smoking, drug use, personal hair dye use, frequent fried food intake and frequent use of a microwave.

$\ddagger$ Model 3: further adjustment for BMI.

glioma (OR 0.42, $95 \%$ CI 0.19, 0.94). Such finding was also seen when further adjustment was made for BMI; such that participants in the highest quartile of DDS were $56 \%$ less likely to have glioma compared with those in the lowest quartile (OR 0.44, $95 \%$ CI $0.20,0.97)$. We also found a significant inverse association between AHEI and odds of glioma; such that in the fully adjusted model, participants in the top quartile of AHEI had $74 \%$ lower risk of glioma compared with those in the bottom quartile after taking all potential confounders, including BMI, into account (OR 0.26, $95 \%$ CI 0.12, 0.56).

\section{Discussion}

We found an inverse association between DDS and risk of glioma. This association was obtained after adjusting for potential confounders including socioeconomic characteristic and BMI. Such inverse association was also seen for AHEI either before or after controlling for potential confounders. To the best of our knowledge, this study is the first to examine the association of DDS and AHEI with glioma.

It is well studied that whole diet rather than individual foods or nutrients can better represent overall diet quality and help predicting the association between diet and diseases ${ }^{(31)}$. DDS and AHEI, as indicators of diet quality, were assessed in relation to non-communicable diseases including CVD and some cancers $^{(32-34)}$. Despite this, no observational study has evaluated the association of these dietary indices with brain cancers such as glioma. In the present study, we found that high dietary diversity was associated with decreased odds of glioma. In line with our findings, some studies had shown an inverse association between DDS and risk of cancers. In a case-control study, adherence to a diet with a high diversity, in particular fruit diversity, was associated with a lower risk of bladder cancer ${ }^{(35)}$. In a prospective study, after a mean follow-up of 8.4 years, variety in the consumption of vegetables and fruit combined and of fruit consumption alone were inversely associated with the risk of oesophageal squamous cell carcinoma ${ }^{(36)}$. In contrast, another study failed to find any significant association between diversity in each food group and risk of breast cancer ${ }^{(37)}$. However, in that study dietary diversity was assessed in a single food group and several confounders were not adjusted for to determine the independent association between dietary diversity and breast cancer. The different nature of breast cancer compared with other cancers might also be another reason for different findings on the association between DDS and cancer. Therefore, further studies are needed to shed light on this issue.

The protective association of DDS against glioma might be explained by the high nutrient content of diverse diet. Antioxidants are one of these nutrients. A large number of studies have shown an inverse association between dietary intake of antioxidants and risk of cancer ${ }^{(38-40)}$. This association was also reported for glioma ${ }^{(41)}$. Another feature of diet with a high DDS is its high content of fruits and vegetables which have been shown to be protective against glioma ${ }^{(8)}$. Diets with a high diversity score are usually rich in fibre which helps the body to reduce carcinogens $^{(42)}$.

In the present study, we found that greater adherence to AHEI was associated with decreased odds of glioma. In our earlier study, adherence to DASH diet, known as a high-quality dietary pattern, was associated with $72 \%$ lower risk of glioma $^{(12)}$. Findings of previous studies on the association between AHEI and risk of other cancers were also in line with our findings. In a large population-based study in Germany, after controlling for potential confounders, individuals in the highest category of AHEI were less likely to have colorectal cancer compared with those in the lowest category ${ }^{(43)}$. Another study revealed an inverse association for ovarian cancer ${ }^{(44)}$. In addition, a systematic review and meta-analysis of observational studies showed that high adherence to AHEI was associated with reduced risk of mortality from cancer ${ }^{(45)}$. The inverse association between AHEI and glioma might be explained by high content of fruits and vegetables. As reported in prior studies, fruit consumption and vegetable consumption have a preventive role for incidence of glioma ${ }^{(6,8)}$. In addition, dietary intake of antioxidants, as anticarcinogenic nutrients, is high among people with greater adherence to $\mathrm{AHEI}^{(46,47)}$. High AHEI score is also associated with a lower intake of red and processed meats which is a known risk factor for glioma ${ }^{(9)}$.

This study has several strengths. This was the first study to examine the association of DDS and AHEI with glioma. We controlled for a wide range of confounders in the present study to reach an independent association between the dietary scores and risk of glioma. In addition, patients enrolled in the study were new cases of glioma who had been diagnosed with this malignancy in the past 1 month. This reduces the possibility of changing usual dietary intakes in these patients. It must also 
be kept in mind that the study comes from the under-studied region of the Middle East where available information on dietdisease relations is very limited.

Some limitations should also be considered when interpreting our findings. The first is the case-control design of the study that is subject to several biases including selection and recall bias. In addition, due to study design we cannot confer causality. Although case-control studies are efficient in terms of time and cost, they are highly susceptible to both selection and recall biases. Recall bias, in which cases may recall their past diet differently in the context of their cancer diagnosis, is problematic because dietary assessment occurs after diagnosis. An additional concern for case-control studies is that cases might have altered their diet before diagnosis due to early symptoms of the disease. However, glioma-related symptoms may lead patients to consume healthy foods rather than unhealthy ones. This would result in attenuated estimates. In addition, cases in the present study were selected from hospitals; therefore, identified risk factors may be unique to these hospitals. However, case-control studies are suitable for studying the association of diet with rare disease with long latency period including cancers. In addition, some controls were selected from those who attended to the outpatients' clinics of the hospital. Although controls had no history of any cancer, they might have a disease with high level of oxidative stress which is known risk factor for several chronic diseases, particularly different cancers ${ }^{(48)}$. Nevertheless, this factor cannot affect dietary intakes and consequently dietary data obtained for controls in the present study. To account for recall bias in this study design, we enrolled hospital-based controls to report exposures in a year before enrolment. As with all epidemiological studies that apply FFQ, misclassification of study participants is unavoidable. Although we controlled for several confounders, one cannot exclude the possibility of residual confounding. In addition, as the dietary intakes of Middle Eastern population are different from Western nations, the generalisability of the findings to other populations should be made cautiously.

In conclusion, greater adherence to a diet with a high diversity was associated with decreased odds of glioma. Such finding was also observed for AHEI; such that participants with a greater adherence to the AHEI diet were less likely to have glioma compared with those with the lowest adherence.

\section{Research and policy implications}

Policies to promote adherence to healthy and diverse dietary patterns in general people might help preventing the incidence of glioma. The beneficial effects of such policies on other chronic conditions have earlier been reported.

\section{Acknowledgements}

This research was supported by the Students' Scientific Research Center, Tehran University of Medical Sciences, Tehran, Iran (code: 97-02-61-38468).

M. S., M. M.-S., G. S. and A. E. contributed in conception, design, statistical analyses, data interpretation and manuscript drafting. O. S. and A. E. contributed in data analysis, data interpretation and manuscript drafting. A. E. finalised the manuscript. All authors approved the final manuscript for submission.

The authors declare that there are no conflicts of interest.

\section{References}

1. Morgan LL (2015) The epidemiology of glioma in adults: a "state of the science" review. Neuro Oncol 17, 623-624.

2. Dolecek TA, Propp JM, Stroup NE, et al. (2012) CBTRUS statistical report: primary brain and central nervous system tumors diagnosed in the United States in 2005-2009. Neuro Oncol 14, Suppl. 5, v1-v49.

3. Williams PT (2014) Reduced risk of brain cancer mortality from walking and running. Med Sci Sports Exerc 46, 927-932.

4. Alimohamadi SM, Ghodsi SM \& Ketabchi SE (2008) Epidemiologic patterns of primary brain tumors in Iran. Asian Pac J Cancer Prev 9, 361-362.

5. Chen H, Ward MH, Tucker KL, et al. (2002) Diet and risk of adult glioma in eastern Nebraska, United States. Cancer Causes Control 13, 647-655.

6. Holick CN, Giovannucci EL, Rosner B, et al. (2007) Prospective study of intake of fruit, vegetables, and carotenoids and the risk of adult glioma. Am J Clin Nutr 85, 877-886.

7. Zhou S, Wang X, Tan Y, et al. (2015) Association between vitamin $\mathrm{C}$ intake and glioma risk: evidence from a meta-analysis. Neuroepidemiology 44, 39-44.

8. Li Y (2014) Association between fruit and vegetable intake and risk for glioma: a meta-analysis. Nutrition 30, 1272-1278.

9. Saneei P, Willett W \& Esmaillzadeh A (2015) Red and processed meat consumption and risk of glioma in adults: a systematic review and meta-analysis of observational studies. J Res Med Sci 20, 602-612.

10. Michaud DS, Holick CN, Batchelor TT, et al. (2009) Prospective study of meat intake and dietary nitrates, nitrites, and nitrosamines and risk of adult glioma. Am J Clin Nutr 90, 570-577.

11. Wei Y, Zou D, Cao D, et al. (2015) Association between processed meat and red meat consumption and risk for glioma: a meta-analysis from 14 articles. Nutrition 31, 45-50.

12. Benisi-Kohansal S, Shayanfar M, Mohammad-Shirazi M, et al. (2016) Adherence to the Dietary Approaches to Stop Hypertension-style diet in relation to glioma: a case-control study. Br J Nutr 115, 1108-1116.

13. Hashemi Kani A, Alavian SM, Esmaillzadeh A, et al. (2013) Dietary quality indices and biochemical parameters among patients with non alcoholic fatty liver disease (NAFLD). Hepat Mon 13, e10943.

14. Caswell BL, Talegawkar SA, Siamusantu W, et al. (2018) A 10food group dietary diversity score outperforms a 7 -food group score in characterizing seasonal variability and micronutrient adequacy in rural Zambian children. J Nutr 148, 131-139.

15. Shiao SPK, Grayson J, Lie A, et al. (2018) Predictors of the Healthy Eating Index and glycemic index in multi-ethnic colorectal cancer families. Nutrients 10, E674.

16. Talaei M, Rabiei K, Talaei Z, et al. (2013) Physical activity, sex, and socioeconomic status: a population based study. ARYA Atheroscler 9, 51-60.

17. Merve A, Millner TO \& Marino S (2019) Integrated phenotypegenotype approach in diagnosis and classification of common CNS tumours. Histopathology (In the Press).

18. Esfahani FH, Asghari G, Mirmiran P, et al. (2010) Reproducibility and relative validity of food group intake in a food frequency questionnaire developed for the Tehran Lipid and Glucose Study. J Epidemiol 20, 150-158.

19. Kant AK, Block G, Schatzkin A, et al. (1991) Dietary diversity in the US population, NHANES II, 1976-1980. J Am Diet Assoc 91, 1526-1531. 
20. Kant AK, Schatzkin A \& Ziegler RG (1995) Dietary diversity and subsequent cause-specific mortality in the NHANES I epidemiologic follow-up study. J Am Coll Nutr 14, 233-238.

21. Kennedy E (2008) Putting the pyramid into action: the healthy eating index and food quality score. Asia Pac J Clin Nutr 17, Suppl. 1, 70-74.

22. Kennedy ET, Ohls J, Carlson S, et al. (1995) The healthy eating index: design and applications. J Am Diet Assoc 95, 1103-1108.

23. Akbaraly TN, Ferrie JE, Berr C, et al. (2011) Alternative Healthy Eating Index and mortality over 18 y of follow-up: results from the Whitehall II cohort. Am J Clin Nutr 94, 247-253.

24. Ruder AM, Carreon T, Butler MA, et al. (2009) Exposure to farm crops, livestock, and farm tasks and risk of glioma: the Upper Midwest Health Study. Am J Epidemiol 169, 1479-1491.

25. Morgan LL, Miller AB, Sasco A, et al. (2015) Mobile phone radiation causes brain tumors and should be classified as a probable human carcinogen (2A) (review). Int J Oncol 46, 1865-1871.

26. Willett WC, Howe GR \& Kushi LH (1997) Adjustment for total energy intake in epidemiologic studies. Am J Clin Nutr 65 , 1220S-1228S.

27. Foerster M, Thielens A, Joseph W, et al. (2018) A prospective cohort study of adolescents' memory performance and individual brain dose of microwave radiation from wireless communication. Environ Health Perspect 126, 077007.

28. Brandes AA \& Paris MK (2004) Review of the prognostic factors in medulloblastoma of children and adults. Crit Rev Oncol Hematol 50, 121-128.

29. Barnholtz-Sloan JS, Ostrom QT \& Cote D (2018) Epidemiology of brain tumors. Neurol Clin 36, 395-419.

30. Malmir H, Shayanfar M, Mohammad-Shirazi M, et al. (2018) Patterns of nutrients intakes in relation to glioma: a case-control study. Clin Nutr 21, 31180-31184.

31. Newby PK \& Tucker KL (2004) Empirically derived eating patterns using factor or cluster analysis: a review. Nutr Rev 62 , 177-203.

32. Arem H, Reedy J, Sampson J, et al. (2013) The Healthy Eating Index 2005 and risk for pancreatic cancer in the NIH-AARP study. J Natl Cancer Inst 105, 1298-1305.

33. Aigner A, Becher H, Jacobs S, et al. (2018) Low diet quality and the risk of stroke mortality: the Multiethnic Cohort study. Eur J Clin Nutr 72, 1035-1045.

34. Lim H, Cho G \& Kim S (2012) Evaluation of nutrient intake and diet quality of gastric cancer patients in Korea. Nutr Res Pract 6 , 213-220.

35. Isa F, Xie LP, Hu Z, et al. (2013) Dietary consumption and diet diversity and risk of developing bladder cancer: results from the
South and East China case-control study. Cancer Causes Control 24, 885-895.

36. Jeurnink SM, Buchner FL, Bueno-de-Mesquita HB, et al. (2012) Variety in vegetable and fruit consumption and the risk of gastric and esophageal cancer in the European Prospective Investigation into Cancer and Nutrition. Int J Cancer 131, E963-E973.

37. Ko KP, Kim SW, Ma SH, et al. (2013) Dietary intake and breast cancer among carriers and noncarriers of BRCA mutations in the Korean Hereditary Breast Cancer Study. Am J Clin Nutr 98, 1493-1501.

38. Lucas AL, Bosetti C, Boffetta P, et al. (2016) Dietary total antioxidant capacity and pancreatic cancer risk: an Italian case-control study. Br J Cancer 115, 102-107.

39. Rossi M, Tavani A, Ciociola V, et al. (2016) Dietary total antioxidant capacity in relation to endometrial cancer risk: a case-control study in Italy. Cancer Causes Control 27, 425-431.

40. Vance TM, Wang Y, Su LJ, et al. (2016) Dietary total antioxidant capacity is inversely associated with prostate cancer aggressiveness in a population-based study. Nutr Cancer 68, 214-224.

41. Mazzio EA \& Soliman KF (2004) Glioma cell antioxidant capacity relative to reactive oxygen species produced by dopamine. J Appl Toxicol 24, 99-106.

42. Zeng H, Lazarova DL \& Bordonaro M (2014) Mechanisms linking dietary fiber, gut microbiota and colon cancer prevention. World J Gastrointest Oncol 6, 41-51.

43. Erben V, Carr PR, Holleczek B, et al. (2018) Dietary patterns and risk of advanced colorectal neoplasms: a large population based screening study in Germany. Prev Med 111, 101-109.

44. Qin B, Moorman PG, Kelemen LE, et al. (2017) Dietary quality and ovarian cancer risk in African-American women. Am J Epidemiol 185, 1281-1289.

45. Onvani S, Haghighatdoost F, Surkan PJ, et al. (2017) Adherence to the Healthy Eating Index and Alternative Healthy Eating Index dietary patterns and mortality from all causes, cardiovascular disease and cancer: a meta-analysis of observational studies. J Hum Nutr Diet 30, 216-226.

46. Vitale E, Jirillo E \& Magrone T (2014) Correlations between the Youth Healthy Eating Index, body mass index and the salivary nitric oxide concentration in overweight/obese children. Endocr Metab Immune Disord Drug Targets 14, 93-101.

47. Weinstein SJ, Vogt TM \& Gerrior SA (2004) Healthy Eating Index scores are associated with blood nutrient concentrations in the third National Health and Nutrition Examination Survey. $J$ Am Diet Assoc 104, 576-584.

48. Saha SK, Lee SB, Won J, et al. (2017) Correlation between oxidative stress, nutrition, and cancer initiation. Int JMol Sci 18, E1544. 